\title{
Active quasioptical Ka-band rf pulse compressor switched by a diffraction grating
}

\author{
O. A. Ivanov, ${ }^{1,2}$ A. A. Vikharev, ${ }^{1}$ A. M. Gorbachev, ${ }^{1,2}$ V. A. Isaev, ${ }^{1}$ M. A. Lobaev, ${ }^{1}$ A. L. Vikharev, ${ }^{1,2}$ S. V. Kuzikov, ${ }^{1}$ \\ J. L. Hirshfield, ${ }^{2,3}$ and M. A. LaPointe ${ }^{3}$ \\ ${ }^{1}$ Institute of Applied Physics RAS, Nizhny Novgorod, Russia \\ ${ }^{2}$ Omega-P, Inc., New Haven, Connecticut 06511, USA \\ ${ }^{3}$ Department of Physics, Yale University, New Haven, Connecticut 06520, USA
}

(Received 29 May 2009; published 11 September 2009)

\begin{abstract}
Results of numerical calculations and experimental investigations of an active Ka-band microwave pulse compressor are presented. The compressor is based on a running-wave, three-mirror, quasioptical resonator utilizing a diffraction grating whose channels embody plasma discharge tubes that constitute the active switch. The principle of compression is based on rapidly changing the resonator's output coupling coefficient ( $Q$ switching) by initiating plasma discharges in the grating channels. Excitation of the resonator was achieved with up to $250 \mathrm{~kW}$ of $34.29 \mathrm{GHz}$ microwaves in $700 \mathrm{nS}$ pulses from the magnicon at the Yale Ka-band Test Facility. A power gain of at least 7:1 in the compressed pulse with a pulse duration of $10-15$ ns was achieved.
\end{abstract}

DOI: 10.1103/PhysRevSTAB.12.093501

PACS numbers: 84.40.Dc

\section{INTRODUCTION}

Two methods for obtaining powerful microwave pulses are passive and active pulse compression [1,2]. An important application of pulse compression systems is for driving accelerator structures. In particular, linear accelerators are recognized as requiring peak microwave power levels that cannot be generated by current state-of-the-art microwave tubes [3].

A number of $\mathrm{rf}$ pulse compression systems have been considered for collider use, including versions of the Delay Line Distribution System and the Resonant Delay Line Pulse Compression System SLED-II [4-6]. The mechanisms upon which these systems operate are passive, in that no element in the microwave circuit has time-dependent properties. The $\mathrm{rf}$ pulse compression in SLED-II is achieved using $180^{\circ}$ phase switching in the microwave generators. Among drawbacks of such systems, one can list a relatively low power gain (less than 4:1) at high compression efficiency and rather large dimensions. It is well known that power gains up to 9:1 can be achieved in passive systems without losses, but as the gain is pushed up the compression efficiency falls dramatically. Moreover, in practice the SLED-II system has microwave losses which reduce the compression efficiency. For example, at a power gain of 4.88 , an efficiency of $24.4 \%$ is realized in practice [2].

A possible way to achieve high microwave peak power is the method of active pulse compression [7,8]. The method is based on storing of energy in a high- $Q$ microwave resonator, and then rapidly increasing the coupling between the resonator and the load ( $Q$ modulation) using a fast switch. This method could find applications due to the possibility of obtaining high power gains $(\sim 8-11: 1)$, with a small sized storage resonator $(\sim 1 \mathrm{~m})$. The availability of high peak-power mm-wave pulses formed through use of active rf pulse compression will expand the parameter range for basic studies of high field effects on material surfaces, and possibly lead to new concepts and designs for mm-wave high-gradient accelerator structures.

This paper describes an active compressor based on a three-mirror traveling-wave resonator operating in the millimeter wavelength band. In such a resonator, the third mirror, which couples the input and output lines and changes the resonator $Q$ factor, is a diffraction grating with parameters that vary when plasma is produced in the grating grooves. Results are presented of calculations and testing for an active quasioptical (QO) compressor excited by magnicon pulses at a frequency of $34.29 \mathrm{GHz}$.

\section{DESIGN AND CALCULATION OF AN ACTIVE QUASIOPTICAL COMPRESSOR WITH A DIFFRACTION GRATING}

An active microwave compressor comprises a storage resonator and a switch, i.e., an element which changes its properties with time. Compressors based on oversized storage resonators, specifically, mirror-type ones, have some advantages. Namely, high selectivity and low loss of operating oscillations can be achieved in a quasioptical resonator. In this case, the switch becomes distributed, which allows reducing the field on the switch to avoid microwave breakdowns. At the same time, to ensure a high $Q$ factor of the cavity, the switch should not cause a significant loss in the energy storage regime and should ensure a short switching time (shorter than the duration of the output pulse) in the regime of energy release.

The distributed switch is based on an electrically controlled diffraction grating (Fig. 1). Essentially, this concept is that an incident wave beam is split into multiple beams differing in their intensities and directions. A change in the 


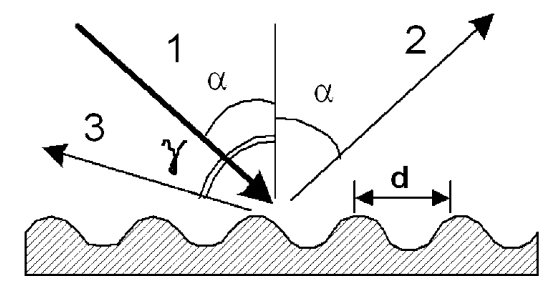

FIG. 1. Splitting of the wave beam incident on the diffraction grating (1) into two, mirror (2) and diffraction (3) beams.

grating parameters changes the ratio of the intensities of the beams reflected from the grating.

For a grating which splits the incident beam into only two beams, namely, the mirror beam and the $(-1 \mathrm{st})$ diffraction beam, the grating period is determined by the following formula:

$$
d=\frac{\lambda}{\sin \alpha+\sin \gamma},
$$

where $\lambda$ is the length of the electromagnetic wave, $\alpha$ is the mirror reflection angle, and $\gamma$ is the angle of reflection into the $(-1 \mathrm{st})$ diffraction maximum.

The scheme of an active QO compressor with an open storage resonator and a beam-switching diffraction grating is shown in Fig. 2. The open three-mirror traveling-wave resonator is formed by the diffraction grating and two microwave focusing mirrors.

In the energy storage regime of the resonator, the main portion of the incident-beam power is reflected specularly from the grating, whereas the balance of the power is branched into the diffraction beam and couples the resonator and the waveguide line [Fig. 2(a)]. Power distribution in the mirror and diffraction beams depends on the grating geometry. When the parameters of the diffraction grating change, the main part of the stored power goes into the diffraction beam and escapes from the resonator [Fig. 2(b)]. If the grating is made as a set of hollow rectangular gas-discharge channels (grooves) in a dielec-

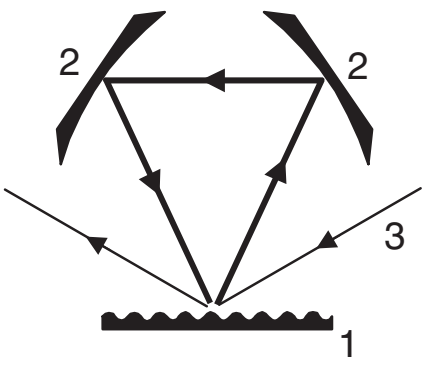

a)

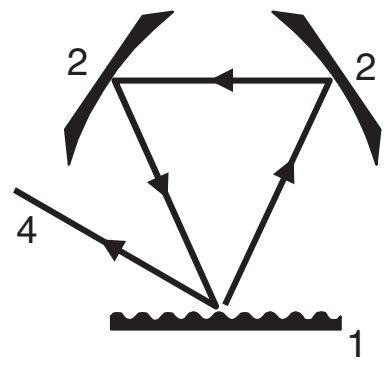

b)
FIG. 2. Compression scheme based on the quasioptical resonator: 1-diffraction grating/switch; 2-focusing mirrors; 3 input pulse; 4-output pulse; (a)—resonator in the regime of energy storage; (b) - resonator in the regime of output of the compressed pulse. tric plate (e.g., quartz) fixed over a flat metal mirror, it can be switched by producing plasma in its grooves. By optimizing the profile and material of the grating, one can achieve high sensitivity of the switch to plasma initiation and reduce the fields on the grooves.

Operation of such a switch was simulated numerically by the finite difference time domain (FDTD) method $[9,10]$, which allows modeling the plasma and its interaction with the electromagnetic field and ensures high calculation accuracy. The computational model of the diffraction grating as a periodic structure over a flat metal mirror is shown in Fig. 3. When this method is used, the finite-difference (network) approximation of time and space derivatives ensures the accuracy $o\left(\Delta t^{2}\right)$ and $o\left(\Delta z^{2}\right)$, where $\Delta t$ and $\Delta z$ are time and space increments, respectively. We considered the case when all plane waves (incident, specularly reflected, and diffraction) lay in one plane, so that the problem could be solved with a twodimensional approximation. The electric-field vector was parallel to the grating grooves (TE polarization). The chosen polarization ensured high electric strength of the switch and efficient influence of the plasma on the electromagnetic waves reflected from the grating.

In the problem under consideration, two waves are reflected from the periodic structure: a mirror wave $I_{0}$ and a diffraction wave $I_{-1}$. In the case of a plane wave, this problem is infinite along the grating plane. To solve it in a finite integration domain, we introduced cyclic boundary conditions, which meant that, in the range of integration, integral numbers of grating periods and periods of mirror $(n)$ and diffraction $(m)$ beams should fit along the groove at the same time. It follows from this condition that the ratio

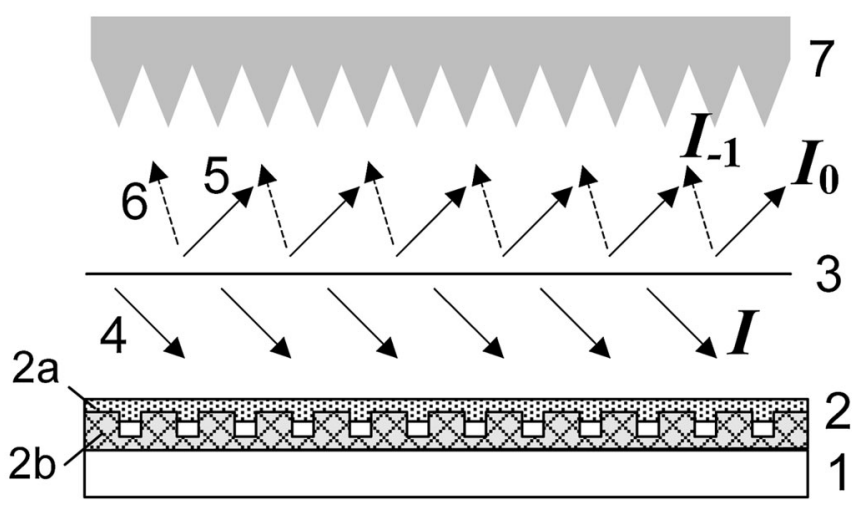

FIG. 3. Computational model of the active diffraction grating: 1-flat metal mirror, 2-dielectric plate with grooves $(2 \mathrm{a}-$ quartz, 2b-Teflon), 3-plane with preset currents, which radiates the wave towards the grating, 4-wave incident on the grating, 5-reflected wave, 6-diffraction wave ( -1 st maximum), 7-absorber (a medium with conductivity that grows linearly in the direction of wave propagation for absorption of the waves reflected from the diffraction grating). 


$$
\frac{\sin \alpha}{\sin \gamma}=\frac{n}{m}
$$

is a rational number. The chosen condition cannot be fulfilled exactly for arbitrary angles $\alpha$ and $\gamma$, but for a larger range of integration (increasing $n$ and $m$ ), it can be fulfilled to any accuracy. A system of electric and magnetic currents was set up in the source plane, such that the currents interacted with only one (incident) wave in the system. This current system emitted a wave at a certain angle in the direction of the grating only (4 in Fig. 3). The intensities of the mirror beam and the $(-1 \mathrm{st})$ diffraction beam reflected from the grating were calculated by expanding the field over plane waves. The waves reflected from the grating were absorbed in the volume with conductivity increasing in the direction from the grating ( 7 in Fig. 3), and the increasing conductivity profile was chosen such that the absorber reflection ratio did not exceed $0.1 \%$. The influence of the plasma was taken into account by introducing conduction currents into the Maxwell's equations:

$$
\begin{gathered}
\nabla \times \vec{E}=-\frac{1}{c} \frac{d \vec{H}}{d t} ; \quad \nabla \times \vec{H}=\frac{\varepsilon}{c} \frac{d \vec{E}}{d t}+\frac{4 \pi}{c} \vec{j} ; \\
\frac{d \vec{j}}{d t}=\frac{e^{2}}{m} N_{e} \vec{E}-\nu(N) \vec{j},
\end{gathered}
$$

where $N_{e}$ is the electron density, $\vec{j}$ is the density of the current in the plasma, $N$ is the density of nitrogen molecules in $\mathrm{cm}^{-3}, \nu(N)=1.7 \times 10^{-7} \mathrm{~N}=$ const, $s^{-1}$ is the characteristic rate of electron-molecule collisions, $c$ is the speed of light, $e$ and $m$ are the charge and mass of the electron, and $\varepsilon$ is the dielectric permeability of the material of the grating. In the calculations, we used a standard grid for the FDTD method. The spacing of the grid was chosen in the range from $0.01 \lambda$ to $0.03 \lambda$, which ensured the necessary accuracy and high speed of the calculation.

Note that when the grating employs a dielectric plate (e.g., quartz one) as the third mirror of the resonator (Fig. 2), the power loss ratio for reflections from the grating is a small value,

$$
\alpha_{L}=\frac{h \varepsilon^{\prime} \omega \operatorname{tg}(\delta)}{c} \approx 0.6 \%,
$$

where $h$ is the grating thickness, $\omega$ is the circular microwave frequency, $\varepsilon^{\prime}$ is the real part of the dielectric permeability of the grating material, $\operatorname{tg}(\delta) \approx 3 \times 10^{-4}$ is the Ohmic-loss ratio, and $c$ is the speed of light. Correspondingly, the $Q$ factor of the resonator caused by such a loss will be

$$
Q=\frac{\omega L}{c \alpha_{L}}=1.2 \times 10^{5},
$$

where $L$ is the path length around three mirrors. One can see that such a loss in the dielectric does not degrade the $Q$ factor of the storage resonator significantly.
Several types of dielectric gratings with various dielectric permeabilities and Ohmic-loss ratios (polystyrol, quartz, ceramics, Teflon) were considered. Calculations showed that the use of homogeneous materials having low Ohmic loss, but sufficiently high dielectric permeability (quartz, $\varepsilon^{\prime}=3.8$; ceramics, $\varepsilon^{\prime} \geq 10$ ) leads to a distortion of the wave front of the wave beam reflected from the grating and, correspondingly, a significant increase in the diffraction loss in the cavity. This is caused by the fact that, when the optical thickness $L_{g}=\sqrt{\varepsilon^{\prime}} \cdot h$ of the grating is great, multiple reflections in the dielectric layer result in formation of a complex structure of the electromagnetic field, which leads to a distortion of the angular spectrum of the Gaussian wave beam which is incident on the grating. Therefore, based on calculations, we chose a composite grating formed by dielectric plates of quartz and Teflon $\left[\varepsilon^{\prime}=2, \operatorname{tg}(\delta) \approx 10^{-4}\right]$ with grooves machined in one of the plates as in Fig. 3. Such grating had a low loss level and ensured a minimal distortion of the wave front of the Gaussian beam reflected from the grating. The dependence of the mirror reflection coefficient for plane waves with incidence angle $\alpha$ for a purely quartz grating and a composite grating is shown in Fig. 4.

It is seen from the figure that, for a purely quartz grating, one observes significant aberrations in the angular spectrum of the plane waves forming the wave beam.

The parameters of the grating were chosen so as to maintain the regime of resonator excitation in the absence of plasma, i.e. a small portion of the incident power was branched into the resonator. When the plasma was produced, the power was directed mainly into the diffraction beam and radiated from the resonator. When modeling the grating switching process, we assumed a plasma with some finite electron density was produced "instantaneously" in

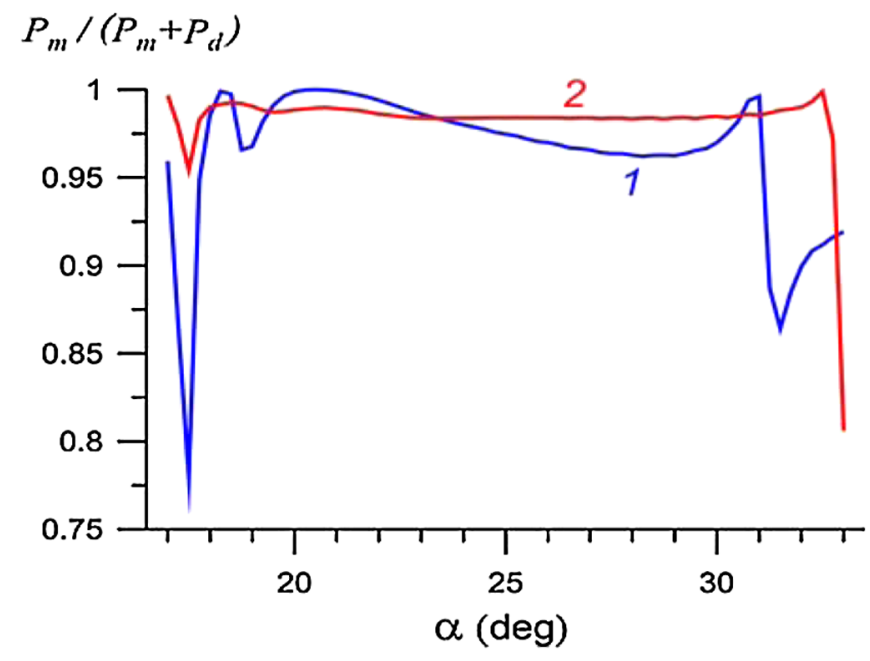

FIG. 4. (Color) Ratio of reflection of a plane wave into a mirror beam is shown as a function of the angle of incidence for quartz and composite gratings ( 1 and 2 , respectively). Here, $P_{d}$ is the power in the diffraction beam and $P_{m}$ is the power in the mirror beam. 

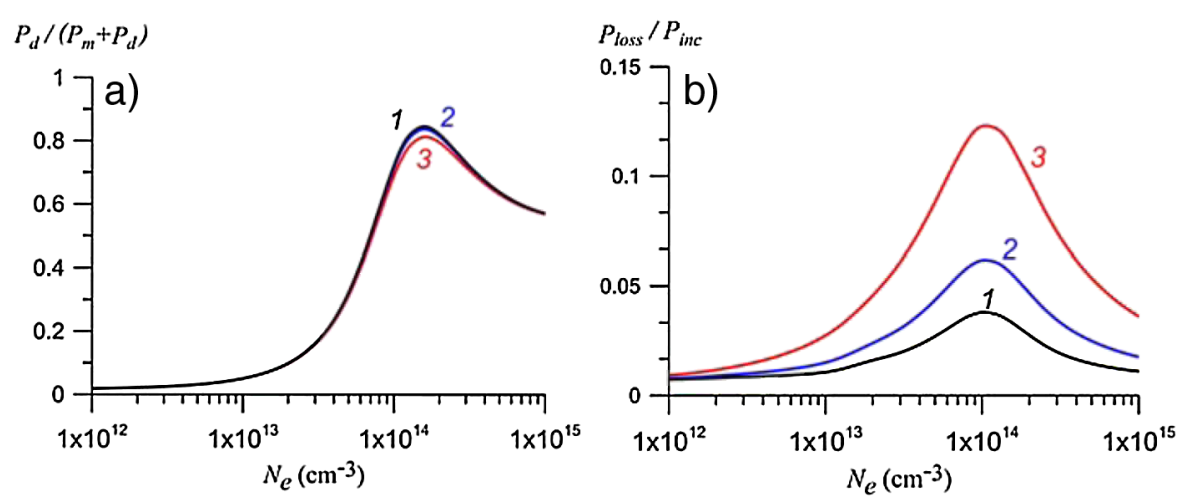

FIG. 5. (Color) (a) Coefficient of reflections from the grating into the diffraction beam as a function of electron density. (b) Share of the microwave power absorbed in the plasma as a function of electron density for different gas pressures. Gas pressure in the grating grooves is 0.3 Torr (1), 1 Torr (2), and 3 Torr (3). Here, $P_{d}$ is the diffraction beam power, $P_{m}$ is the mirror beam power, $P_{\text {loss }}$ is the power absorbed in the plasma, and $P_{\text {inc }}=P_{d}+P_{m}+P_{\text {loss }}$ is the incident power.

the gas-discharge channels with the gas having a preset density. Gas density and electron density in the channels of grating were varied in the calculation process.

Figure 5(a) shows the calculated coefficient of reflection into the diffraction beam as a function of electron density for an electromagnetic-wave frequency of $34.29 \mathrm{GHz}$, angles of the incident and diffraction beams equal to $25^{\circ}$ and $60^{\circ}$, respectively, and different gas density in the grating grooves. Figure $5(\mathrm{~b})$ shows the share of the microwave power absorbed in the plasma produced in the grooves as a function of electron density and gas pressure.

The calculations showed that, for efficient switching, the electron density should exceed the critical value $N_{e}>$ $10^{13} \mathrm{~cm}^{-3}$. Specifically, when a plasma with electron densities ranging from $10^{13}$ to $10^{14} \mathrm{~cm}^{-3}$ is produced in the grating, the share of the microwave power branched into the diffraction beam (the switching coefficient) changes from $8 \%$ to $70 \%$. It was necessary to use low gas pressures $p<3$ Torr to reduce the absorption of microwaves in the produced plasma. In this case, the Ohmic loss in the plasma does not exceed $10 \%$. Note also that the typical switching time is determined by the rate of gas ionization in the gasdischarge grooves and, by estimations, does not exceed $10 \mathrm{~ns}$.

Thus, the calculations show the possibility of creating a switch based on a diffraction grating which has the required parameters, namely, a low branching ratio in the storage regime and a high ratio in the energy release regime.

\section{EXPERIMENTAL STUDY OF DIFFRACTION GRATING AND ACTIVE QO PULSE COMPRESSOR}

Based on the above calculations, a diffraction grating with gas-discharge grooves was manufactured. The grating was made of two plates, one of quartz and the other of Teflon. In the $(240 \times 120) \mathrm{mm}^{2}$ Teflon plate, hollow rectangular grooves were made, which communicated on one side to pump the working gas in and out of the formed channels. The cross section of each channel was $(3.6 \times$ 2.3) $\mathrm{mm}^{2}$, and 29 channels formed the grating with a period of $6.79 \mathrm{~mm}$, which was fixed flush to a plane metal mirror. At both ends of the grooves, metal electrodes were glued in. Thus, each channel had a high-voltage stainlesssteel electrode which received a negative pulse with an amplitude of 50-100 kV and a duration of $100 \mathrm{~ns}$ (the rise time was less than $10 \mathrm{~ns}$ ) through a limiting resistor $R=$ $1 \mathrm{k} \Omega$. The grating was connected with the vacuum system via a special vacuum port, so that the pressure of the nitrogen in the grating grooves could be changed without disrupting the vacuum in the chamber. When a highvoltage pulse was fed in, the gas in the channels broke down and a gas discharge was initiated.

The density of the produced plasma was estimated based on the value of the current running through the grating grooves. The total current through all discharge channels was measured by a noninductive probe. The maximum current amounted to $J_{\max }=600-700$ A, i.e., a current of $J_{\mathrm{CH}} \sim 40$ A ran through each channel for the grating comprised by 18 channels. This current was used to estimate plasma density in the grating grooves:

$$
N_{e}=\frac{J_{\mathrm{CH}}}{e S V_{d}},
$$

where $S=7.8 \mathrm{~mm}^{2}$ is the transverse section of the plasma channel, $V_{d}=3.38 \times 10^{6}(E / p)^{0.5}$ is the electron drift velocity in nitrogen for the values of the electric field and pressure in the range $120<E / p<3000 \mathrm{~V} /$ (cm-Torr) [11], $E=U / L_{P}$, and $L_{p}$ is the length of the plasma channel. Estimations show that the density of electrons is close to the critical value $N_{C}=m \omega^{2} / 4 \pi e^{2}$ or exceeds it in a rather wide range of nitrogen pressures $p=0.3-10$ Torr (for an electromagnetic wave of $34 \mathrm{GHz}$, the critical density is $N_{C}=1.46 \times 10^{13} \mathrm{~cm}^{-3}$ ). Plasma density grows with pressure monotonically and amounts to $1.8 \times$ $10^{13} \mathrm{~cm}^{-3}$ or $2.8 \times 10^{13} \mathrm{~cm}^{-3}$ for pressures of 0.6 and 7 Torr, respectively. Indeed, the maximum electron density 


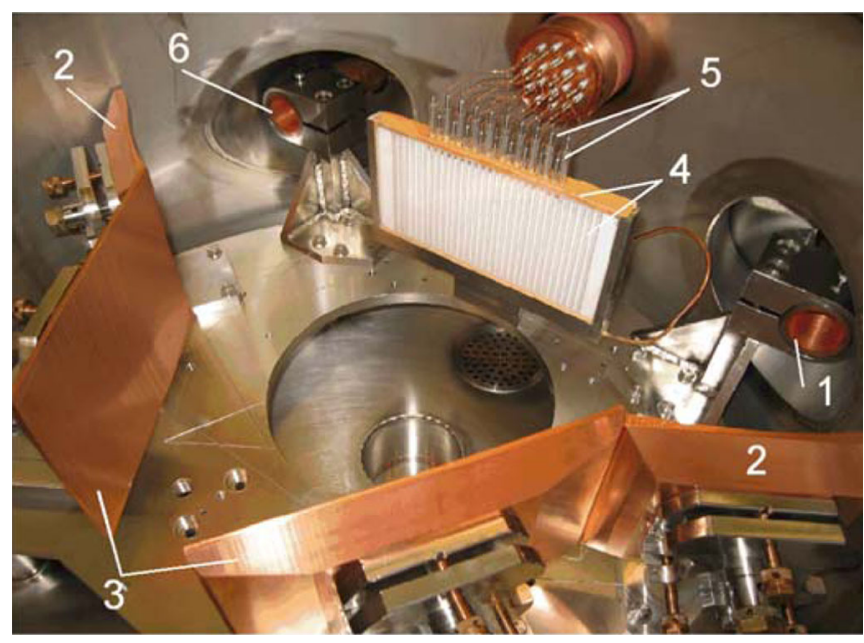

FIG. 6. (Color) The three-mirror resonator arrangement for the QO compressor with a diffraction grating: 1-input horn, 2focusing mirrors provide the coupling from the Gaussian mode launchers to the resonator through the secondary lobe of the grating, 3-resonator mirrors, 4-active diffraction grating, 5electrodes, 6-output horn.

is somewhat higher than the calculated value due to nonuniform distribution of plasma density over the cross section of the discharge channel. Specifically, at higher pressures, a streamer discharge with an extremely narrow cross section starts. However, in this case the discharge stability is much poorer.

Next, the operation of a plasma diffraction grating acting as a switching component of an active QO compressor was studied. The general view of the quasioptical three-mirror resonator with an electrically controlled diffraction grating is shown in Fig. 6.

The radiation was input to and output from the resonator by means of horns (1) and (6) and focusing mirrors (2). The resonator was formed by two copper mirrors (3) and a diffraction grating (4) comprised by a dielectric plate and a plane metal mirror. Pulsed voltage produced by a highvoltage generator was fed to the electrodes of diffraction grating (5) through a set of ballast resistors. The compressor was pumped out by ion pumps down to the pressure of $10^{-6}-10^{-7}$ Torr.

The frequency characteristic of the three-mirror cavity was measured using a scalar network analyzer (HP model 8757D). Measurements showed that the loaded $Q$ factor $Q_{L}=Q_{0} Q_{e} /\left(Q_{0}+Q_{e}\right)$ of the cavity is $Q_{L}=5.4 \times 10^{4}$ for the frequency $f_{0}=34.29 \mathrm{GHz}$ and the parameter $\beta=$ $Q_{0} / Q_{e}$ (where $Q_{0}$ is the inherent $Q$ factor for the cavity, and $Q_{e}=k L / \alpha_{e}$ is the coupling $Q$ factor, $k=2 \pi / \omega$, $\alpha_{e}$-coupling coefficient, $L$ resonators length) reached a value of $\beta=0.65-0.75$, while the transmission of microwaves in the mirror beam approached $90 \%$. Note that these values differ somewhat from the parameters $\left(Q_{L}=3.6 \times\right.$ $\left.10^{4}, \beta=1.2-1.3\right)$ that are optimal for the excitation of the resonator with microwave pulses $600-700 \mathrm{~ns}$ long.

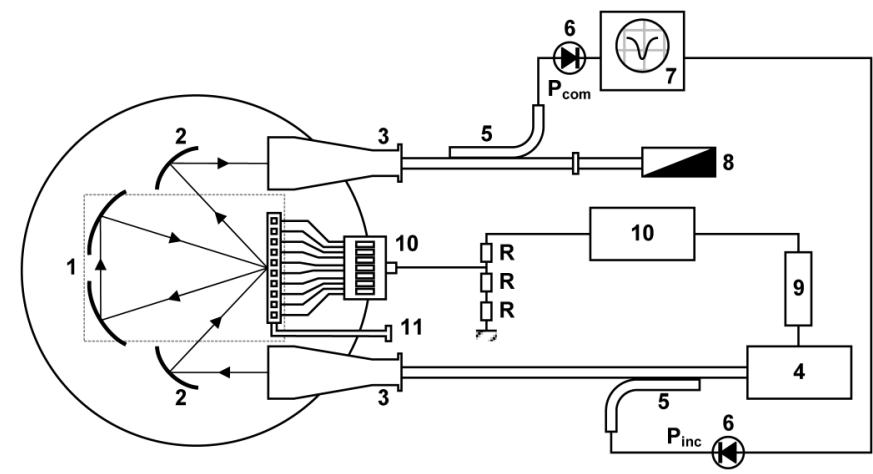

FIG. 7. Scheme of experimental setup of quasioptical microwave compressor with a diffraction grating: 1-three-mirror cavity, 2-focusing mirror, 3-input and output horns, 4magnicon, 5-directional coupler, 6-detector, 7-oscilloscope, 8-load, 9-delay line, 10-high-voltage pulse generator and cutoff resistor unit, 11-diffraction grating evacuation unit.

Next, the power storage regime in the QO compressor was studied without switching the high-voltage pulse generator (HVPG). The scheme of the experiment is shown in Fig. 7. Excitation of the storage resonator (1) was achieved with up to $250 \mathrm{~kW}$ of $34.29 \mathrm{GHz}$ microwaves in $700 \mathrm{nS}$ pulses from the magnicon (4). The output pulse of the compressor went to a matched load (8) connected to the output horn (3). The signals proportional to the input and output powers, $P_{\text {inc }}$ and $P_{\text {com }}$, were sent to the detectors (6) through directional couples (5) with coupling coefficients of -54 and $-48 \mathrm{~dB}$, respectively. The signals from the detectors were recorded with a digital oscilloscope (7).

Tuning the compressor to the magnicon operation frequency was achieved by varying the length of the threemirror resonator. The tuning quality was checked by minimizing the output-signal power $P_{\text {com }}$ with the restriction that the difference between the output signal out of resonance and on resonance remained at the maximum. Characteristic oscillograms of the compressor output sig-

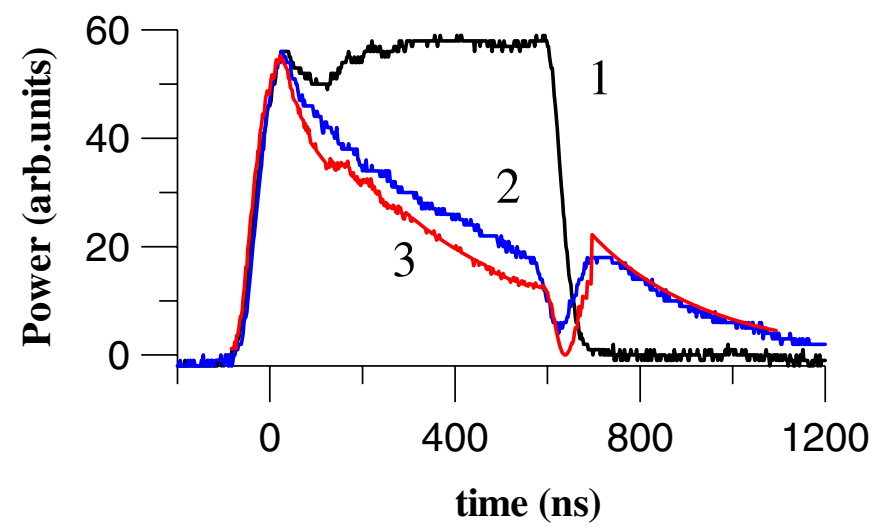

FIG. 8. (Color) Traces from the output of the QO compressor show the nonresonant case (1-experiment) and a resonant case (2-experiment, 3 - calculation for resonator parameters: $Q_{L}=$ $\left.5.4 \times 10^{4}, \beta=0.65\right)$. 


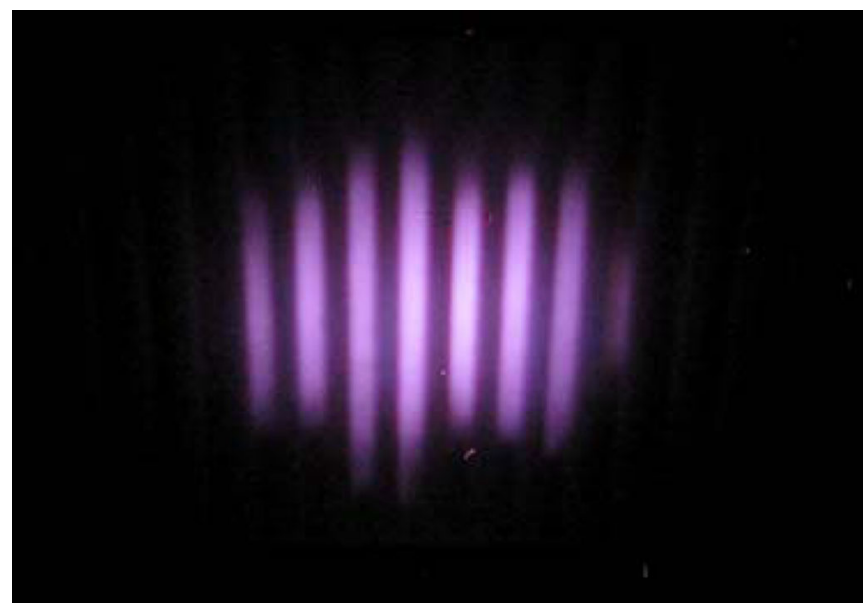

FIG. 9. (Color) Microwave breakdown inside diffraction grating (distance between plasma layers is equal $6.9 \mathrm{~mm}$ ).

nal $P_{\text {com }}$ out of resonance (curve 1 ) and on resonance (curve 2) are shown in Fig. 8. It is seen that on resonance after the magnicon pulse (curve 2), $P_{\text {com }}$ decreases exponentially, which allows one to obtain the $Q$ factor of the loaded cavity: $P_{\text {com }} \propto \exp (-t / \tau)$, where $\tau=Q_{L} / \omega$. Note also that the results of calculating the compressor output signal in resonance (curve 3 ) for the experimentally measured profile of the magnicon impulse and the parameters of the storage resonator $Q_{L}=5.4 \times 10^{4}$ and $\beta=0.65$ agrees with the experimental data (curve 2) with reasonable accuracy.

The difference between output signals in the nonresonant and resonant case represents the energy stored in the compressor. It follows from Fig. 8 that for the measured parameters, the value of the injected energy reaches $35 \%-$ $40 \%$, when the pulse duration of the magnicon is about 600 ns. Lower excitation efficiency is related to the high $Q$ factor $Q_{L}$ and, correspondingly, a narrower width of the resonance curve compared with the width of the magnicon pulse spectrum. Moreover, in some magnicon operating regimes, a minor frequency deviation was observed during the microwave pulse, which leads to a reduction of the efficiency of energy storage in the resonator. Therefore, in the experiments small modifications to the magnicon op- eration (such as magnetic field magnitude and profile, trim fields near the cathode, input power levels, etc.) were made so as to minimize this frequency deviation.

At the same time, the breakdown characteristics of the active diffraction grating were determined. Experiments showed that for the input pulse power $P_{\text {inc }} \sim 250 \mathrm{~kW}$, a microwave breakdown happens in grating grooves when the pressure exceeded 5-6 Torr of air. In this case, the value of the electric field produced in the grating grooves by the incident wave amounts to $E_{i}=1.57 \mathrm{kV} / \mathrm{cm}$ out of resonance and $E_{r}=12.2 \mathrm{kV} / \mathrm{cm}$ in resonance. Note that for the resonance case the power of the wave incident on the grating reaches $P_{r} \sim 16 \mathrm{MW}$. The photo of a microwave discharge in the grating is shown in Fig. 9.

Next the QO compressor was studied for the case of simultaneous gas breakdowns in the channels of the diffraction grating. The compressor was switched from the microwave power storage regime into the regime of power output by means of the HVPG (10 in Fig. 7). The HV pulser was placed in a shielding box and connected to the compressor via an inductance free divider limiter $(R)$ that provided the voltage of 50,75 , or $100 \mathrm{kV}$ to the gasdischarge channels. A high-voltage pulse with duration $50 \mathrm{~ns}$ was fed to the each electrode of the active grating via a limiting $1000 \mathrm{Ohm}$ resistor. The pressure of air (or nitrogen) in the grating for these experiments was changed in the range $p=0.3-10$ Torr. The trigger pulses went to the modulator of the magnicon and the delay generator (9). From the delay generator the trigger pulse was sent to the HVPG. This synchronization scheme made it possible to control the timing of the HVPG pulse with respect to the microwave pulse.

Experiments showed that uniformity of the DC discharge in the grating channels grew significantly with the amplitude of the high-voltage pulse. Figure 10 shows photos of the discharge in the grating under pressure $p=$ 1.5 Torr for the amplitudes of the high-voltage pulse equal to 50 and $100 \mathrm{kV}$, respectively.

Experiments with active pulse compression were then performed. A high-voltage pulse was fed into the grating grooves and a plasma was produced. The coefficient of the grating reflection into the -1 st diffraction maximum grew from $1.5 \%$ to $10 \%-20 \%$ and the stored energy was released
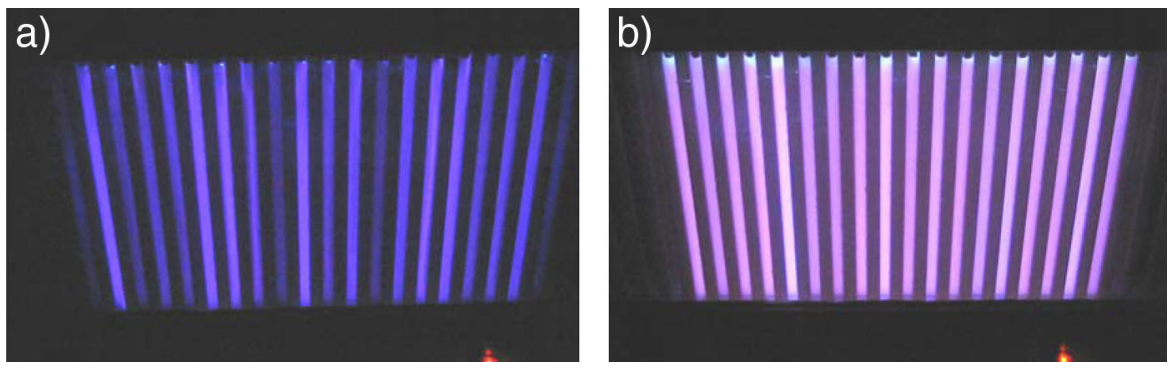

FIG. 10. (Color) High-voltage discharge inside diffraction grating: (a) amplitude of high-voltage pulse $50 \mathrm{kV}$; (b) amplitude of highvoltage pulse $100 \mathrm{kV}$, distant between plasma layers is equal $6.9 \mathrm{~mm}$. 

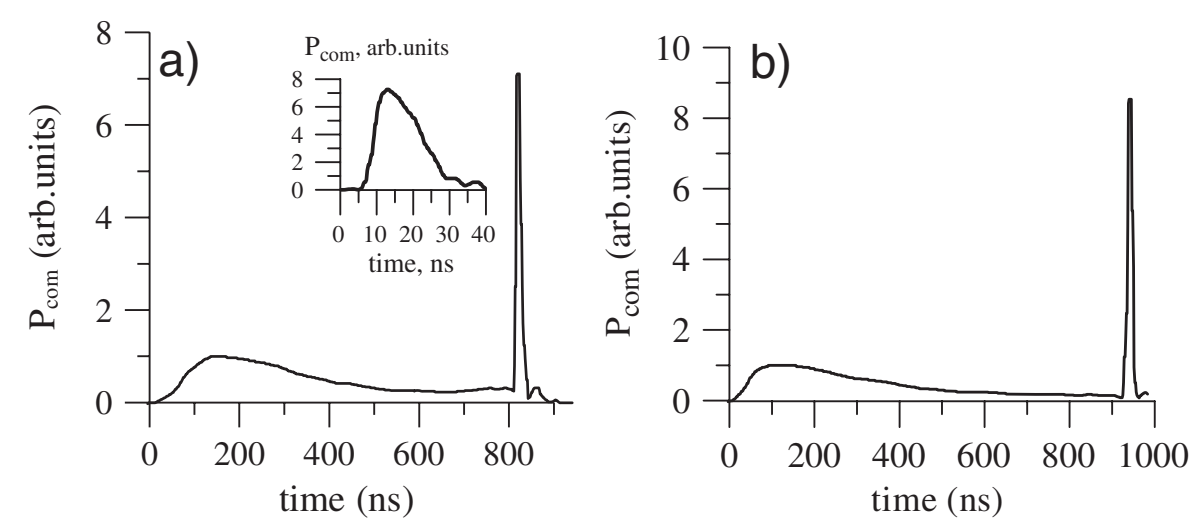

FIG. 11. Characteristic oscillograms of the compressed pulse in the output of the quasioptical active pulse compressor.

from the resonator to the output channel of the compressor in 5-7 transits of the resonant circuit. As a result a compressed pulse $P_{\text {com }}$ was observed in the output channel of the compressor. The characteristic oscillograms of the compressed pulses are shown in Fig. 11. The amplitude and duration of the compressed pulses depended on the gas pressure in the grating with the best results obtained in the range of air pressures from 0.7 to 2 Torr. Under the excitation of the resonator with $250 \mathrm{~kW}$ of $34.29 \mathrm{GHz}$ microwaves in 600-700 ns pulses from the magnicon, a power gain of 7 in the compressed pulse with duration of 10-20 ns was achieved. By tentative estimations, total compression efficiency amounted to $25 \%$. It should be noted that longer magnicon pulses (up to $900 \mathrm{~ns}$ ) resulted in higher energy stored in the resonator and hence a higher-power gain ratio [Fig. 11(b)].

The compression efficiency stated above was defined as the ratio of the energy in the compressed pulse to the energy in the incident pulse. The energy in the compressed pulse was calculated by integrating the pulse envelope over the time intervals where the amplitude is greater than $10 \%$ of the maximum. The energy in the incident pulse was also calculated by integrating the pulse envelope over the duration of the pulse except during the rise time where a frequency deviation was observed. The low efficiency obtained was due to the fact that for the parameters used in the storage resonator the switching time of the grating was comparable with the propagation time of the wave around the three mirrors $\tau_{r}$. The stored energy was extracted during 5-7 $\tau_{r}$ but during the switching time the stored energy was not extracted and was lost. To increase the compression efficiency, as well as to obtain a rectangular shape of the compressed pulse, it is necessary to increase the path length around the three mirrors. From our previous experiment at low power level [12], it was shown that after plasma generation the phase of the reflected wave from the diffraction grating is stable on the order of $1000 \mathrm{~ns}$ which is defined by plasma decay in the grating grooves. This latter fact is important for linear collider applications of the active quasioptical compressor.
Thus, the experiments have demonstrated the principal of using an active diffraction grating for compression of microwave pulses and energy output from a high- $Q$-factor quasioptical resonator operated in the millimeter wavelength band.

\section{DISCUSSION AND CONCLUSION}

Calculations show that the maximum electric field $E_{\mathrm{Ch}}$ in the gas-discharge channel equals approximately the amplitude $E_{r}$ of the electromagnetic-wave incident on the grating in the resonator, $E_{\mathrm{Ch}}=1.02 E_{r}$. However, the electric field $E_{s}$ on the grating surface is significantly lower and amounts to $E_{s}=0.42 E_{r}$. Hence, the electric strength of the compressor is limited primarily by the microwave breakdown of the gas in the grating grooves. Figure 12 shows the time dependence of the amplitude of the electric field in the grating grooves in the energy storage regime for an incident pulse power $P_{\text {inc }}=250 \mathrm{~kW}$.

It follows from the figure that for input pulse duration of $700 \mathrm{~ns}$ the value of the electric field in the grooves reaches $12 \mathrm{kV} / \mathrm{cm}$ at the end of the accumulation stage. The power incident on the grating amounts to $16 \mathrm{MW}$.

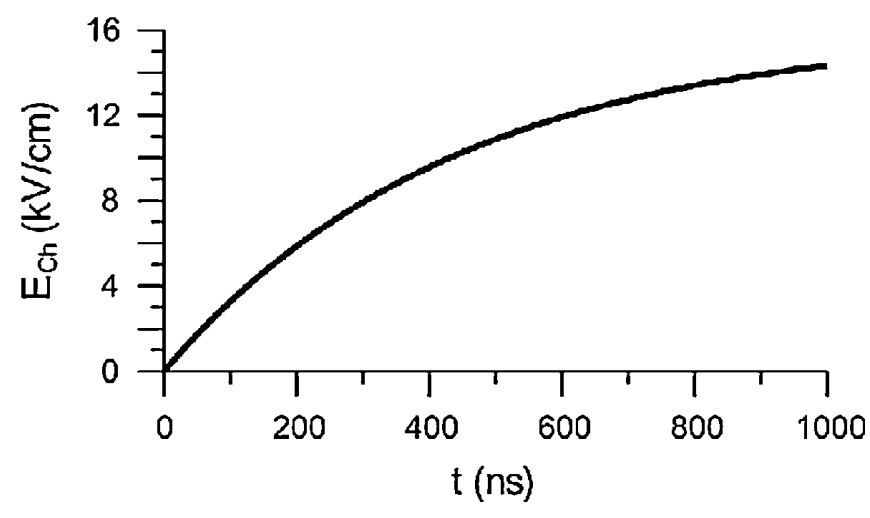

FIG. 12. Time behavior of the maximum amplitude of the electric field in a grating groove in the energy storage regime in the resonator for the incident power $P_{\text {inc }}=250 \mathrm{~kW}$. 
The maximum input power of the compressor can be determined by calculating the electric field at which a microwave breakdown of the gas in the grating occurs. In turn, breakdown fields can be estimated by the electron balance equation assuming that the ionization is local and the electron loss is determined by diffusion in the pressure range where $\nu<\omega$ :

$$
\frac{d N_{e}}{d t}=\left\{\nu_{i}\left[E_{e}(t), p\right]-D / \Lambda^{2}\right\} N_{e} .
$$

Here, $\nu_{i}\left[E_{e}(t), p\right]$ is the rate of electron-impact ionization, which depends on the effective electric field $E_{e}=$ $E(t) \nu /\left(\omega^{2}+\nu^{2}\right)^{1 / 2}, E(t)$ is the electric field in the resonator (Fig. 12), $\nu$ is the rate of electron-molecule collisions, $D=1.6 \times 10^{6} / p\left[\mathrm{~cm}^{2} / \mathrm{s}\right]$ is the diffusion coefficient, $\Lambda^{-2}=\left(\pi / d_{1}\right)^{2}+\left(\pi / d_{2}\right)^{2}$ is the diffusion length [13], $d_{1}$ and $d_{2}$ are transverse dimensions of a diffraction grating groove, and $p$ is the gas pressure. The dependence $\nu_{i}\left[E_{e}(t), p\right]$ from [14] was used in the calculations.

Figure 13(a) shows the time evolution of microwave breakdown (i.e., the time during which the electron density reaches the critical level $N_{C}$ ) in a channel as a function of the gas pressure for two different values of the input pulse power from the magnicon. The results of calculating the maximum power of a $700 \mathrm{~ns}$ input pulse for the compressor as a function of the air pressure are shown in Fig. 13(b).

It follows from the figures that for a preset duration of the magnicon pulse, the power of the input pulse, at which no microwave breakdown occurs in the grating, grows considerably as the gas pressure decreases. At the same time, to ensure fast and efficient switching of the grating to the energy release regime, the gas pressure should not be much lower than $p=1$ Torr. The experiments showed that the electron density in a high-voltage discharge which is required for the switching, specifically $N_{e} \geq 10^{13} \mathrm{~cm}^{-3}$, is reached in the pressure range $p \geq 0.7-1.5$ Torr. The maximum power of the input pulse which corresponds to these pressures amounts to $P_{\text {inc }} 1.5-3.5 \mathrm{MW}$.
Thus the peak power of compressed pulse is limited by microwave breakdown in the channels of the diffraction grating and is defined by certain parameters: $Q$ factor of the storage resonator, gas pressure in the channels of the grating, and transverse dimensions of the wave beam on the grating. For the parameters used in this storage resonator, the power density threshold in the wave beam on the grating, corresponding to an incident power $P_{\mathrm{inc}} \approx$ $3.5 \mathrm{MW}$, is equal to $0.4 \mathrm{MW} / \mathrm{cm}^{2}$.

To increase the power of the input pulse further, it is necessary to decrease the amplitude of the electric field on the grating. Such a decrease can be achieved by increasing the cross-section area of the wave beam on the grating or by decreasing the coupling $Q$ factor of the storage resonator. Estimations show that for the nitrogen pressure in the grooves $p=0.6-1$ Torr a twofold increase in the area of the cross section of the Gaussian beam results in an increase of the maximum power of the input pulse up to $P_{\text {inc }}=5-6 \mathrm{MW}$. Then for the same power density of $0.4 \mathrm{MW} / \mathrm{cm}^{2}$ the power of a compressed pulse with a duration of 20-25 ns will be up to $P_{\text {com }}=50-70 \mathrm{MW}$.

The following conclusions are made based on the results of experiments performed. (i) It is shown that a selfbreakdown in the grooves of the diffraction grating (with pressure $p>5$ Torr) occurs when the power of the wave incident on the grating exceeds $16 \mathrm{MW}$. Because of an increase of the diffusion loss the decrease in the pressure in the grating leads to a significant increase in the electric strength of the diffraction grating and the possibility to compress higher-power microwave pulses. (ii) The compressed pulses obtained in the experiments had peak powers of 1.6-1.9 MW and durations of 10-20 ns. In this case the power gain was at least $k=7$. Calculated efficiency of the compression in the experiments was $25 \%$. (iii) Experiments showed that, when the magnicon pulse duration is $\sim 700 \mathrm{~ns}$, one has to make the coupling parameter $\beta$ equal to 1.2-1.5 in order to increase the efficiency of energy storage and output pulse power. (iv) A novel plasma switch based on diffraction grating for an active Ka-band
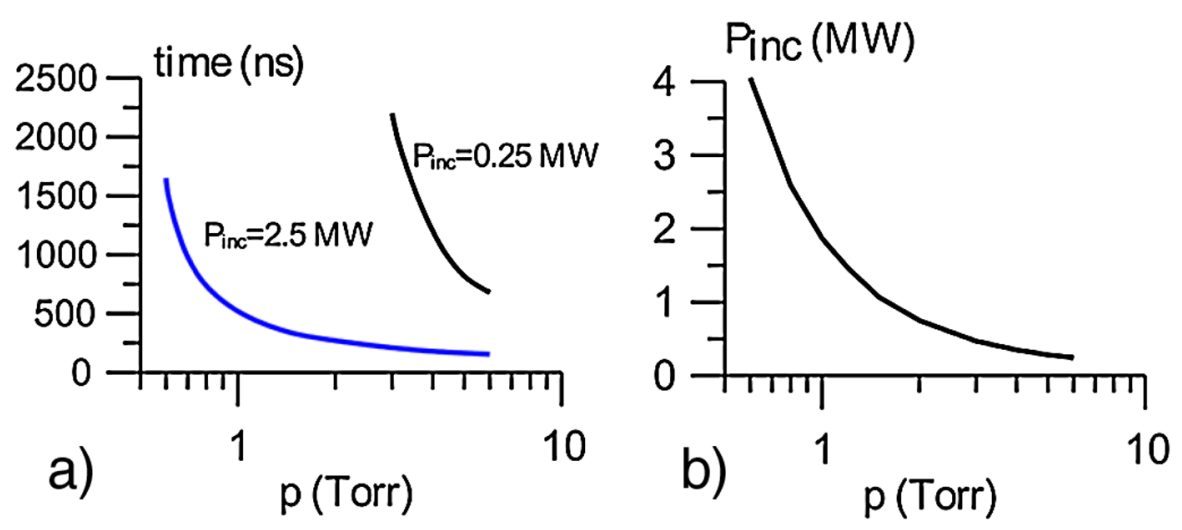

FIG. 13. (Color) (a) Breakdown evolution time as a function of air pressure for different incident powers $P_{\text {inc }}$. (b) Maximum power of the input pulse as a function of air pressure in the grating (input pulse duration $t=700 \mathrm{~ns}$ ). 
pulse compressor has been developed. A compressor with a new optimized switch should be able to provide 50$70 \mathrm{MW}, 20 \mathrm{~ns}$ output pulses at $34 \mathrm{GHz}$. The availability of $\mathrm{mm}$-wave rf pulses in this power range will expand the parameter range for basic studies of high-field interactions with material surfaces, and to possible development of novel high-gradient mm-wave accelerator structures.

\section{ACKNOWLEDGMENTS}

This work was supported by the U.S. Department of Energy, Office of High Energy Physics.

[1] R. Alvarez et al., Part. Accel. 11, 125 (1981).

[2] P. B. Wilson, in Applications of High-Power Microwaves, edited by A. V. Gaponov-Grekhov and V.L. Granatstein (Artech House, Boston-London, 1994), Chap. 7.

[3] P. B. Wilson, Z. D. Farkas, and R. D. Ruth, in Proceedings of the Linear Accelerator Conference, Albuquerque, NM (SLAC Report No. SLAC-PUB-5330, 1990).

[4] P. B. Wilson, in Proceedings of the Conference on Pulsed RF Sources for Linear Colliders, Montauk, Long Island, New York, 1994.

[5] H. Mizuno and Y. Otake, in Proceedings of the 17th International Linear Accelerator Conference (LINAC94), Tsukuba, Japan, 1994 (KEK, Japan, 1994) (KEK Report No. 94-112, 1994).
[6] S. G. Tantawi and C. D. Nantista, Recent advances in RF Pulse Compressor Systems at SLAC, High Energy Density and High Power RF, 6th Advanced Accelerator Concepts Workshop, edited by S. H. Gold and G. S. Nusinovich, AIP Conf. Proc. No. 691 (AIP, Melville, New York, 2003), p. 172.

[7] A. L. Vikharev, A. M. Gorbachev, O. A. Ivanov, V. A. Isaev, S. V. Kusikov, J. L. Hirshfield, O. A. Nezhevenko, S.H. Gold, and A.K. Kinkead, Radiophys. Quantum Electron. 46, 802 (2003).

[8] A. L. Vikharev, A. M. Gorbachev, O. A. Ivanov, V. A. Isaev, S. V. Kusikov, M. A. Lobaev, J. L. Hirshfield, S. H. Gold, and A. K. Kinkead, Phys. Rev. ST Accel. Beams 12, 062003 (2009).

[9] K.S. Yee, IEEE Trans. Antennas Propagat. AP-14, 302 (1966).

[10] A. Taflove, Advances in Computational Electrodynamics. The Finite-Difference Time-Domain Method (Artech House, Boston-London, 1998), p. 724.

[11] A. W. Ali, Laser Part. Beams 6, 105 (1988).

[12] A. L. Vikharev, A. A. Vikharev, A. M. Gorbachev, O. A. Ivanov, V. A. Isaev, and S. V. Kusikov, Tech. Phys. Lett. 35, 421 (2009).

[13] Y.P. Raizer, Gas Discharge Physics (Springer, Berlin, 1987).

[14] A. V. Gurevich, N. D. Borisov, and G. H. Milikh, Physics of Microwave Discharges: Artificially Ionized Regions in the Atmosphere (Gordon and Breach, Amsterdam, 1997). 